\title{
Capacidad de rebrote de Leucaena macrophylla Benth con fines dendroenergéticos en Cortes, Honduras
}

\author{
Regrowth capacity of Leucaena macrophylla Benth for dendroenergy purposes in \\ Cortes, Honduras
}

\author{
Daniel Abisai Flores-Pinot ${ }^{-}$Thelma Janeth-Sorto ${ }^{2}$ - Javier Gutiérrez-Bardales ${ }^{3} \bullet$ Dagoberto Arias-Aguilar $^{4}$ (D) • \\ Juan Carlos Valverde ${ }^{4}$ (iD Jesús Mora- Molina ${ }^{5}$
}

\section{Abstract}

The effect of cutting season, cutting height and sowing density in the management of Leucaena macrophylla Beneth regrowths was evaluated. We used an experimental plantation in Cortés, Honduras. The forest crop was

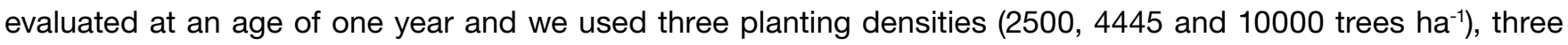
pruning treatments were applied within the design (control, 40 and $100 \mathrm{~cm}$ stump height), which were applied in the dry (March 2016) and rainy (July 2017). After the regrowth was cut, the mortality, type of regrowth, diameter growth and the height of the regrowth after one year were evaluated. The data revealed that there is no difference in the regrowth mortality rate as a function of the pruning season (average of $57.34 \%$ ), besides that regardless of the spacing and pruning height, the epicormic regrowth was the one that presented the highest presence. Regarding the diametric growth, variations were recorded between 1.2 and $4.3 \mathrm{~cm}$ and the variations in height from 0.5 to $5.8 \mathrm{~m}$; In addition, a higher growth rate was found in the rainy season (15 to $35 \%$ more than the dry season). No differences were found between the pruning at 40 and $100 \mathrm{~cm}$ and finally it was determined that a density of 1000 trees ha ${ }^{-1}$ negatively affected the growth of the plantation and generated growth decreases up to $20 \%$ with respect to the densities of 4445 and 2500 trees ha $^{-1}$, which did not show differences between them.

Key words: Biomass, pruning, harvest, growth, cutting season, sprouts, bioenergy.

1. Consultor Independiente; Francisco Morazán, Honduras; floresdaniel335@yahoo.com

2. Docente UNACIFOR; Comayagua, Honduras; tjaneth_sorto@hotmail.com

3. Departamento de Energéticos y Medio Ambiente, INCAL S.A de C.V; San Pedro Sula, Honduras; jgutierrezb@calidra.com.mx

4. Escuela de Ingeniería Forestal, Tecnológico de Costa Rica; Cartago, Costa Rica; darias@tec.ac.cr; jcvalverde@tec.ac.cr 


\section{Resumen}

Se evaluó el efecto de la época de corta, altura de corta y densidad de siembra en el manejo de rebrotes de árboles de Leucaena macrophylla Beneth, para lo cual se utilizó una plantación experimental en Cortés, Honduras. El cultivo forestal se evaluó a una edad de un año y se establecieron tres densidades de siembra (2500, 4445 y 10000 árb ha-1), dentro del diseño se aplicaron tres tratamientos de poda (testigo, 40 y 100 $\mathrm{cm}$ de altura de tocón), los cuales fueron aplicados en la época seca (marzo 2016) y lluviosa (julio 2017). Posterior a la corta del rebrote, se evaluó la mortalidad, tipo de rebrote, crecimiento diamétrico y la altura del rebrote transcurrido un año. Los datos revelaron que no hay diferencia en la tasa de mortalidad de rebrotes en función de la época de poda (promedio del 57,34\%), además que indiferentemente del espaciamiento y altura de poda, el rebrote de tipo epicórmico fue el que presentó mayor presencia. Con respecto al crecimiento diamétrico se registraron variaciones entre 1,2 a 4,3 $\mathrm{cm}$ y la variaciones en altura de 0,5 a 5,8 m; además se encontró una tasa de crecimiento mayor en la época lluviosa (del 15 a $35 \%$ más que la época seca). No se encontraron diferencias entre la poda a 40 y $100 \mathrm{~cm}$ y finalmente se determinó que una densidad de 1000 árb ha ${ }^{-1}$ incidió negativamente en el crecimiento de la plantación y generó disminuciones de crecimiento hasta del $20 \%$ con respecto a las densidades de 4445 y 2500 árb ha-1 ${ }^{-1}$ que no mostraron diferencias entre sí.

Palabras clave: Biomasa, poda, cosecha, crecimiento, época de corta, rebrotes, bioenergía.

\section{Introducción}

Se estima que en los próximos 50 años la demanda energética mundial se incrementará en un $32 \%$ debido al crecimiento de la población mundial, aumento de la automatización de líneas de producción industrial, uso de transporte eléctrico y crecimiento de las economías de los países en vías de desarrollo [1]; además se prevé que para el 2050 el $52 \%$ de la energía implementada procederá de fuentes renovables, esto como producto de políticas de uso de las energía renovables y los avances en la investigación y desarrollo, encarecimiento de los sistemas de producción energético no renovable y aumento en la dificultad de obtención de fuentes energéticas tradicionales (combustibles fósiles, gas natural, entre otros de bajo impacto ambiental) [2]. Por lo que el uso de las energías alternativas se convierte en una de las líneas de investigación de mayor relevancia para la próxima década [3].
Entre las energías renovables que han mostrado mayor potencial de uso a nivel mundial y con énfasis en la región Latinoamericana, la biomasa se constituye como una fuente muy prometedora de origen vegetal, Brown et al. [4] destaca tres aspectos sobre el uso de la biomasa: i. simplicidad de obtención (se puede transformar a partir de residuos de los sistemas productivos o producir a partir de plantaciones con altas densidades de siembra), ii. Baja contaminación ambiental en su obtención (la cosecha de biomasa requiere tecnologías simples, con impacto ambiental bajo en comparación a otras fuentes como combustibles fósiles o carbón mineral) y iii. fuente de generación cíclica (un cultivo puede generar varios ciclos de cosecha de biomasa en el tiempo, permitiendo optimizar las áreas productivas). Baettig et al. [5] mencionan que la bioenergía a partir de la biomasa puede ser utilizada en regiones latinoamericanas en las que se disponen de áreas superiores a 5 millones de hectáreas de terreno sin un uso definido sin causar deforestación, con costos de transporte energético elevado y limitaciones tecnológicas que inciden que la población no disponga electricidad o su coste sea elevado.

En Honduras, el $59 \%$ del consumo eléctrico es dedicado a las actividades industriales y apenas el $32 \%$ es implementado para un uso doméstico [6] se estima que el $49 \%$ de los hogares hondureños utilizan intensivamente biomasa (específicamente leña), para sustentar las actividades domésticas y el 21,8 \% de la población combina la biomasa con electricidad y gas natural para actividades hogareñas [7]. A partir del 2008 el Gobierno Hondureño ha desarrollado una estrategia de diversificación de la matriz energética, en la que se ha estimulado el desarrollo de micro redes energéticas a partir de biomasa [8]. En varios países de la región centroamericana, los gobiernos han definido prioridades para uso y desarrollo de fuentes de biomasa estables para el mediano plazo, que permitan generar un mercado bioenergético formal, con estándares claros al momento de transformar la materia prima [9]. El establecimiento y manejo de plantaciones dendroenergéticas se convierte en una de las opciones más factibles para el mercado bioenergético hondureño; Lu et al. [2] mencionan que el cultivo de árboles a alta densidad de siembra permite generar altos volúmenes de biomasa en ciclos cortos (de 2 a 4 años), además de la utilización de terrenos bajo nuevos sistemas productivos, aumentando los ingresos económicos a los propietarios (producto a la disminución de terrenos ociosos) e incidiendo positivamente en las economías locales.

Especies árboreas como Leucaena macrophylla muestran gran potencialidad para su uso energético debido a que es una especie de rápido crecimiento, con facilidad de rebrote [11] y con un poder calórico de 
$19000 \mathrm{~kJ} \mathrm{~kg}^{-1}$, superior en comparación a especies como Gmelina arborea (16000 kJ kg-1) o Eucalyptus deglupta $\left(17900 \mathrm{~kJ} \mathrm{~kg}^{-1}\right)$, todas estas especies actualmente se están implementado en proyectos dendroenergético en América Central [10].

En Honduras, las experiencias en dendroenergía han sido limitadas y se han enfatizado en el manejo y utilización de los residuos forestales producto de las plantaciones con fines maderables; por lo que es necesario el desarrollo de nuevas investigaciones sobre el uso de especies bajo condiciones de una plantación dendroenergética y a partir de sus resultados la generación de paquetes tecnológicos para la obtención de biomasa de forma estable. Empresas como INCAL S.A de C.V. han iniciado el desarrollo de los primeros estudios dendroenergéticos en el país, evaluando especies con potencial energético comercial. El presente estudio evaluó el efecto de la época de poda, altura de corte y densidad de siembra para el manejo de los rebrotes de Leucaena macrophylla con fines dendroenergéticos en Potrerillos, Cortes, Honduras.

\section{Materiales y métodos}

\section{Especie y sitio de estudio}

El estudio se realizó en una plantación de 10,67 ha de Leucaena macrophylla Benth, establecida en el municipio de Potrerillos, Cortés $\left(15,166^{\circ} \mathrm{N}, 87,967^{\circ} \mathrm{O}\right)$. El municipio se ubica a 45 kilómetros al sur de la ciudad de San Pedro Sula. La plantación se estableció en un área de uso agrícola y forestal (figura 1).

El sitio se localiza a una altitud de $169 \mathrm{~m}$, con una topografía relativamente plana (una pendiente inferior a $5 \%$ ), con condiciones de suelo de textura francoarenosa; una temperatura promedio anual de $26^{\circ} \mathrm{C}$ y una precipitación anual de $1324 \mathrm{~mm}$ [11]. Previo al cultivo, el sitio se limpió con una chapia manual en la cual se eliminó la cobertura presente y se dejó el suelo desnudo.

Las plántulas de L. macrophylla fueron obtenidas a partir de fuentes semilleras de la especie en Honduras. Las plantas se germinaron en un vivero con condiciones controladas por un periodo de 2 meses bajo un sistema de siembra directa en bolsa plástica de polietileno con un sustrato de $50 \%$ suelo (horizonte A del sitio) y una mezcla con granza de arroz.

En el campo se establecieron tres densidades de siembra: 2500 árb ha-1 $(2,0 \times 2,0 \mathrm{~m}), 4445$ árb ha $^{-1}(1,5 \mathrm{x}$ $1,5 \mathrm{~m})$ y 10000 árb ha-1 $(1,0 \times 1,0 \mathrm{~m})$. Por cada densidad se sembró un área total de 3,56 ha. En el diseño, la ubicación de cada densidad dentro de la finca fue designada de forma aleatoria.

\section{Tratamientos aplicados}

Diez meses después de la siembra de la plantación, en cada una de las densidades evaluadas se montaron ocho parcelas permanentes (24 parcelas en total) de $8 \times 8 \mathrm{~m}$ con una distribución completamente al azar y un error de muestreo inferior al $5 \%$. Cada parcela se dividió en tres subparcelas en las cuales se aplicaron tres tratamientos de poda: i. corta a $40 \mathrm{~cm}$ de altura, ii. corta a $100 \mathrm{~cm}$ (se cortó toda la superficie leñosa de las plantas en alturas superiores a $100 \mathrm{~cm}$ ) y ii. testigo (no se cortaron los árboles). Los tres tratamientos de corta se aplicaron tanto en la época lluviosa (mes de julio del 2016) y seca (mes de enero del 2017 en la que los árboles presentaron una edad de 18 meses); lo anterior con el fin de evaluar el impacto de la época de corta en la capacidad de rebrote.

Los cortes de los rebrotes re realizaron con una tijera podadora de corte bisel, el corte se realizó con un ángulo de $35^{\circ}$ y se revisó que no presentara ningún defecto debido al corte, de tal manera que todo fuera uniforme. La evaluación y clasificación de los rebrotes se realizó dos meses posteriores a la poda y la evaluación de la mortalidad se registró seis meses posterior a la poda.

\section{Variables analizadas}

Se analizó el efecto de la variación climática entre la época seca y lluviosa en el periodo realizado, lo cual complementó con los datos de una estación climática Davis, con mediciones climáticas programadas cada 60 minutos.

En cada parcela se evaluó el diámetro de la base del árbol, para lo cual se utilizó un vernier electrónico marca VINCA de $15 \mathrm{~cm}$ de longitud y la altura se evaluó con una vara telescópica marca Forestry de $8 \mathrm{~m}$ de altura máxima. También se evaluó el tipo de rebrote, para lo cual se utilizó la clasificación que define tres tipos de rebrote [12]:

1. Adventicias: son tejidos formados en callos de cicatrización, generados en el proceso de cicatrización del tocón, y su sistema vascular no proviene directamente del haz del tronco (figura 12a).

2. Epicórmicas: se desarrollan alrededor de la superficie perimetral de tocón; son de origen endógeno y están conectadas con el xilema primario y se mueven hacia fuera en la medida que el árbol crece radialmente (figura 2b). 


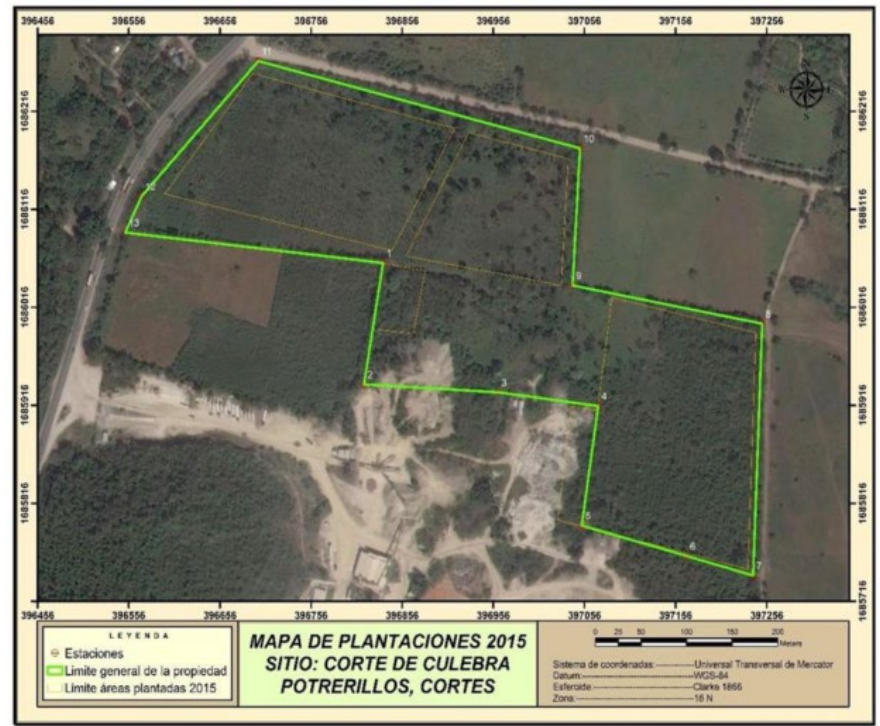

Figura 1. Ubicación del sitio de la plantación de L. macrophylla bajo tres espaciamientos en Cortés, Honduras.

Figure 1. Planting side of $L$. macrophylla with three spacings in Cortés, Honduras.

3. Lignofiber: se forman en la base del tallo, pueden ser numerosas y formar un abultamiento leñoso (figura 2c).

A cada árbol se le clasificó el tipo de rebrote dos meses después de aplicada la poda y posteriormente se evaluó a los cuatro meses post-poda para la estimación de la mortalidad.

\section{Diseño experimental y análisis de resultados}

El experimento se realizó bajo un diseño simple aleatorio. Primeramente, se analizó el tipo de rebrotes y la mortalidad que se obtuvieron con las tres densidades de siembra y la poda realizada en la época seca y lluviosa. Seguidamente se evaluó el efecto en la densidad de siembra y la altura de corte en el crecimiento diamétrico y en la altura en ambas épocas, para lo cual se realizó un análisis de varianza de una vía (ANDEVA) para determinar la existencia de diferencias significativas (con su respectiva comprobación de los supuesto del análisis de varianza: normalidad de los residuos y homogeneidad de las varianzas). Para determinar las diferencias significativas se aplicó la prueba de Tukey con una significancia de 0,05 y la ayuda del programa Infostat versión 2017.

\section{Resultados y discusión}

\section{Variación climática entre la época seca y lluviosa del estudio}

Los datos climáticos mostraron diferencias significativas a nivel de precipitación mensual, la época lluviosa (de mayo a octubre) mostró una precipitación significativamente mayor $\left(75,9 \mathrm{~mm} \mathrm{mes}^{-1}\right)$ con respecto a la seca que fue de $51,1 \mathrm{~mm}$ mes-1; la temperatura no mostró variación significativa entre ambas épocas siendo en promedio de $26,5^{\circ} \mathrm{C}$. COMMEND [6] menciona que el sitio de estudio
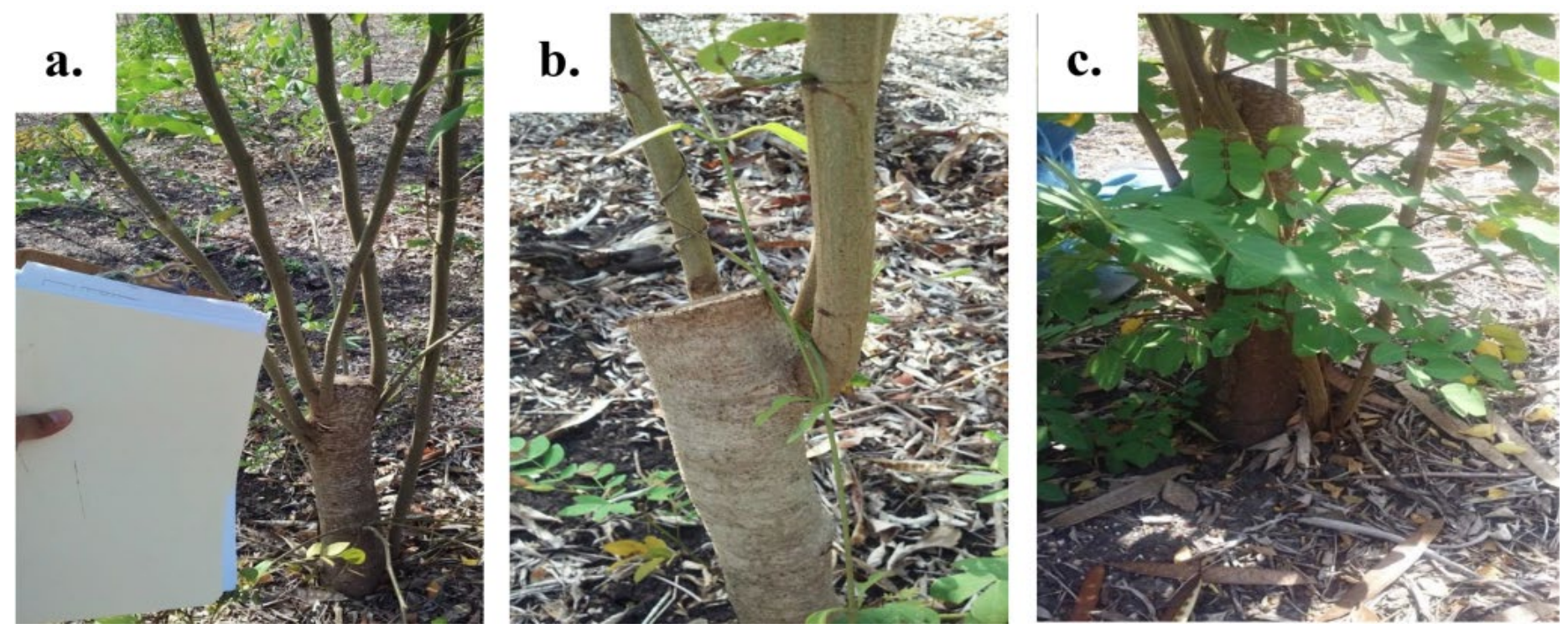

Figura 2. Tipos de rebrotes evaluados adventicias (a), epicórmicos (b) y lignofiber (c), en árboles podados de L. macrophylla.

Figure 2. Types of regrowth evaluated adventitious (a), epicormic (b) and lignofiber (c), on pruned trees of L. macrophylla. 
Cuadro 5. Mortalidad y tipo de rebrote según la época de poda y densidad de siembra en árboles juveniles de L. macrophylla en Cortés, Honduras.

Table 5. Mortality and type of regrowth according to the pruning season and density of sowing in juvenile trees of $L$. macrophylla in Cortés, Honduras.

\begin{tabular}{|c|c|c|c|c|c|}
\hline \multicolumn{6}{|c|}{ Estación lluviosa } \\
\hline \multirow{2}{*}{$\begin{array}{c}\text { Densidad de } \\
\text { siembra (árb/ha) }\end{array}$} & \multirow{2}{*}{ Mortalidad (\%) } & \multicolumn{4}{|c|}{ Porcentaje de rebrotes } \\
\hline & & Adventicios & Epicórmicos & Lignofiber & Total \\
\hline 10000 & \multirow{3}{*}{58,57} & 4,06 & 95,94 & - & 100,00 \\
\hline 4445 & & - & 89,41 & 10,59 & 100,00 \\
\hline 2500 & & 2,02 & 97,97 & - & 100,00 \\
\hline \multicolumn{6}{|c|}{ Estación Seca } \\
\hline \multirow{2}{*}{$\begin{array}{c}\text { Densidad de } \\
\text { siembra (árb/ha) }\end{array}$} & \multirow{2}{*}{ Mortalidad (\%) } & \multicolumn{4}{|c|}{ Porcentaje de rebrotes } \\
\hline & & Adventicios & Epicórmicos & Lignofiber & Total \\
\hline 10000 & \multirow{3}{*}{56,10} & 1,03 & 98,97 & - & 100,00 \\
\hline 4445 & & 10,91 & 89,01 & - & 100,00 \\
\hline 2500 & & 6,13 & 93,87 & - & 100,00 \\
\hline
\end{tabular}

presenta normalmente una demarcación entre la época seca y lluviosa en la que la precipitación se disminuye entre un 25 y $34 \%$.

\section{Efectos de la época de poda en la mortalidad y tipo de rebrotes}

Los resultados de las evaluaciones de las parcelas no mostraron diferencias significativas en cuanto al porcentaje de mortalidad a nivel de rebrote entre las dos épocas evaluadas (cuadro 1); en promedio se obtuvo una mortalidad del $57,34 \%$, valor considerado como aceptable según menciona Singhal et al. [13] para las especies de género Leucaena sp. La alta mortalidad de rebrotes se debe a que la planta al ser podada entra en estrés fisiológico lo que tiende a generar nuevos ejes en mayor cantidad, con el fin de restablecer los procesos de fotosíntesis y transpiración. Una vez generada la nueva cobertura foliar, tiende a eliminar los ejes con menor grado de desarrollo o de menor aporte energético (la generación de azúcares por producto de la fotosíntesis es baja), lo cual incide que la mortalidad aumente de un 40 a $60 \%$.

No se encontraron diferencias entre ambas épocas climáticas (seca y lluviosa). Esto se explica por el estado juvenil de las plantas. Nassar et al. [14] determinó para Leucaena greggii de 7 años de edad, una tasa de mortalidad del $44 \%$ en época seca y $61 \%$ en época lluviosa, la diferencia generada se debió a que en época lluviosa existe mayor presencia en el ambiente de patógenos (bacterias, virus y hongos) lo cual aumenta la infestación y muerte de los rebrotes.
En cuanto a la cantidad de rebrotes generados (cuadro 1), se determinó que la época seca generó una cantidad significativamente mayor (4,68 rebrotes/planta), con respecto a la estación lluviosa (1,78 rebrotes/planta). La diferencia encontrada concuerda a los estudios de Reda et al. [15] con especies arbóreas perennes tropicales, encontrando que un aumento de los rebrotes en época seca es una respuesta mecánica al estrés hídrico que presenta la planta. Un árbol podado al no tener copa en presencia de déficit hídrico, debe desarrollar una nueva cobertura foliar que permita generación de azúcares y el intercambio gaseoso para la sobrevivencia del individuo. En el caso de la época lluviosa, se dispone de una mayor cantidad de agua, lo cual incide en que la planta genere una menor cantidad de rebrotes pero con un crecimiento mayor debido a la presencia de condiciones más favorables.

Con respecto al tipo de rebrote (cuadro 1), se encontró que el rebrote más común fue del tipo epicórmico, con una presencia promedio del $94,20 \%$, seguido por el tipo adventicio y el lignofiber, este último únicamente se presentó en la época seca para la densidad de siembra de 4445 árb ha-1 $^{-1}$. El dominio de rebrote epicórmico se debe a que es un rebrote de rápido desarrollo, con presencia de meristemos secundarios y en los que el consumo energético es menor en comparación al adventicio o lignofiber que son rebrotes de lento desarrollo y de un consumo energético mayor [15], además el rebrote epicórmico es según Dynesium et al. [12] el más común del género Leucaena sp en regiones tropicales, por lo que indiferentemente de la densidad de siembra, la especie tendería a la generación de dicho tipo de rebrote. 

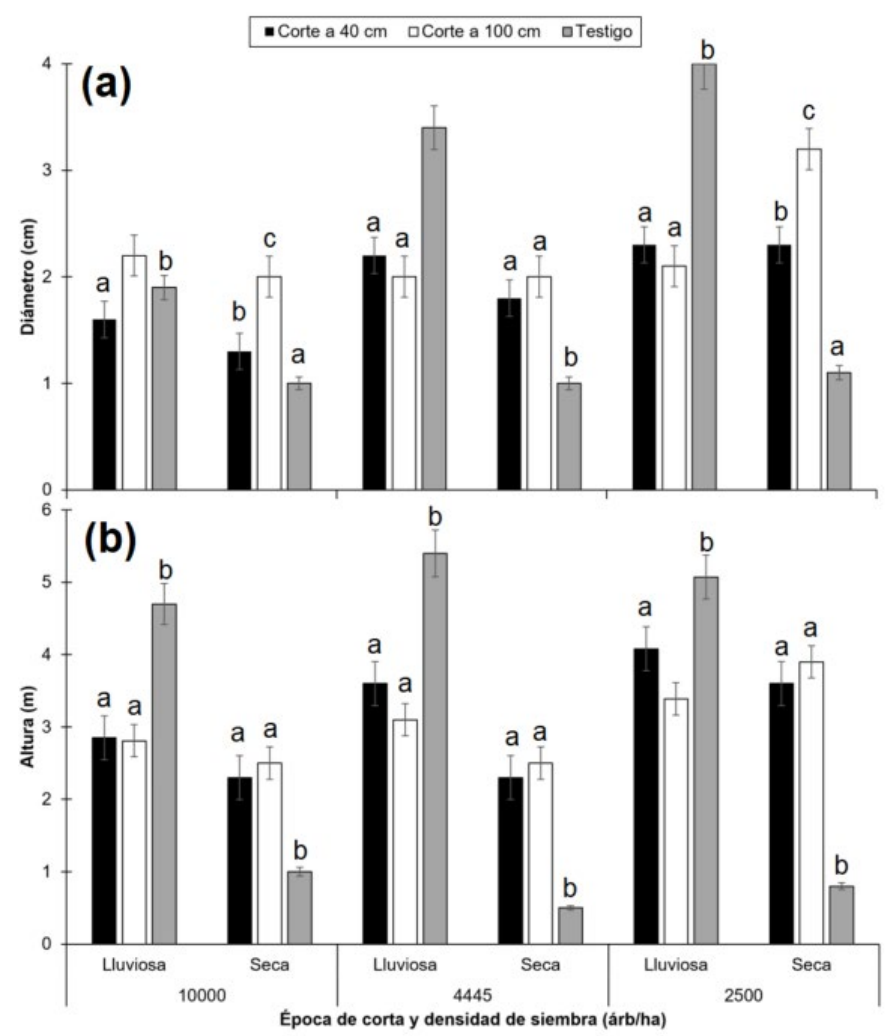

Figura 3. Crecimiento en diámetro (a) y en altura total (b) de árboles de L. macrophylla con tres tipos de poda, establecidos con tres densidades de siembra y cortados en dos épocas del año en Cortés, Honduras.

Figure 3. Growth in diameter (a) and height (b) of $L$. macrophylla trees with three types of pruning, established with three planting densities and cut at two seasons of year in Cortés, Honduras.

\section{Efecto de los tratamientos en la altura y diámetro de los rebrotes}

El crecimiento diamétrico (figura 3a) varió de 1,2 a 4,3 $\mathrm{cm}$, y se encontraron las siguientes características: i. indiferentemente de la poda y la densidad de siembra aplicado, se encontró que el crecimiento en época lluviosa es significativamente mayor (del 10 a $24 \%$ ); ii. se encontró que la densidad de siembra de 10000 árb ha-1 mostró crecimiento diamétrico significativamente menor con respecto a los otros tratamientos; sin embargo, no se encontraron diferencias entre las densidades de 4445 y 2500 árb ha $^{-1}$; iii. no se encontraron diferencias significativas entre la poda de 40 y $100 \mathrm{~cm}$ de altura, pero fueron significativamente mayores al testigo (exceptuando en época lluviosa en la densidad de 4445 y 2500 árb ha-1 $^{-1}$ que el crecimiento de testigo fue mayor).

Con respecto al crecimiento de altura (figura $3 b$ ) varió de 0,5 a 5,8 $\mathrm{m}$ y se encontró que la época lluviosa incidió que el crecimiento fuera de un 20 a $35 \%$ mayor que en la época seca en todos los tratamientos. Igualmente, no se encontraron diferencias significativas entre la poda a 40 y $100 \mathrm{~cm}$ (variaciones entre de 2,1 a 4,5 cm). Finalmente, se determinó que la densidad de 10000 árb ha-1 presentó un crecimiento menor (de 1,1 a 5,2 cm), en comparación a las densidades de 4445 y 2500 árb ha $^{-1}$ (entre ambos no se encontró diferencias estadísticas y presentaron variaciones de entre 0,8 a $6,1 \mathrm{~cm}$.

El crecimiento mayor de diámetro y altura en época lluviosa se debió a que es el momento en que el árbol cuenta con mayor cantidad de agua en el suelo y nutrientes disponibles [12], en el caso de la época seca la disponibilidad de agua en la planta genera que el individuo desarrolle medidas de control hídrico, reduciendo el crecimiento primario y secundario y centrando las actividades metabólicas en sobrevivir al déficit [16]. Por lo que el desarrollo de actividades de poda en época lluviosa (indistintamente la densidad de la plantación o altura de la poda) generó mayor crecimiento, y se obtuvieron tendencias similares a las presentados por Nassar et al. [14] para especies del género Leucaena sp. (crecimiento en época lluviosa del 20 a $30 \%$ en diámetro y del 15 a $25 \%$ en altura).

La ausencia de diferencias entre las dos alturas de poda aplicadas, se debió a la capacidad de rebrote de Leucaena, Dynesium et al. [12] mencionan que la capacidad de $L$. macropylla se da entre los 10 y $230 \mathrm{~cm}$ de altura y en periodos de 45 días post poda presentan la formación de rebrotes con capacidad fotosintética, es una especie con facilidad de rebrote y adaptabilidad a las condiciones de poda (únicamente presenta limitaciones en cortes con rajaduras o afectaciones por patógenos). En el caso del tratamiento testigo, al no aplicarse una operación de poda, se obtuvieron valores mayores en altura y diámetro, debido a que las plantas pueden invertir la energía de recuperación que usan los árboles podados en crecimiento diamétrico y altura, con ello la diferencia se amplió, pero con la limitación que la cantidad de ejes es menor con respecto a los individuos manejados [16].

Finalmente, el efecto de la densidad de siembra mostró

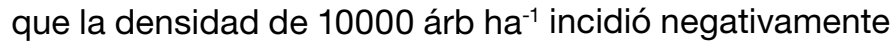
en el crecimiento de los individuos, esto debido a que una alta densidad de siembra (mayor a 3000 árb ha-1) causó que los árboles compitieran por el espacio de luz necesaria para el proceso fotosintético; lo cual a su vez incidió en un estrés negativo (proceso en el que el individuo pierde condiciones de crecimiento y entra en pérdida de desarrollo fisiológico), caracterizado por una copa que generó pocas hojas y con ello la disminución de la fotosíntesis [4], caso contrario con densidades de 4445 y 2500 árb ha ${ }^{-1}$, en las que el individuo contó con un espacio suficiente para crecer, desarrollar una copa competitiva y capaz de realizar la fotosíntesis necesaria para el crecimiento y acumulación de biomasa [5]. Lu et al. [2] mencionan que en plantaciones con fines 
energéticos en los que el ciclo de corta es inferior a tres años, se debe evaluar y optimizar la densidad de siembra ya que altas densidades incidirán en tasas de mortalidad mayores, poca acumulación de biomasa y un costo económico productivo mayor, por lo que definir la densidad de productividad máxima de un cultivo es vital para el éxito de la plantación y disminución del costo de la materia prima. En este estudios, el uso de una densidad de 10000 árb ha-1 , no es la mejor opción para el cultivo del L. macrophylla con fines dendroenergeticos.

\section{Conclusiones}

El estudio encontró que el manejo de rebrotes de la especie L. macrophylla no mostró diferencias en el porcentaje de mortalidad entre la época seca y lluviosa (promedio de 57,34 \%), sin embargo, la época seca mostró una mayor cantidad de rebrotes que en la época lluviosa. En ambos casos se dio una dominancia del rebrote de tipo epicórmico. Con respecto al crecimiento, se obtuvieron variaciones en crecimiento del diámetro entre de 1,2 a 4,3 cm y en altura de 0,5 a 5,8 m; se encontró mayor crecimiento en la época lluviosa (del 15 a 35 \% más que la época seca).

No se encontraron diferencias significativas entre la poda a 40 y $100 \mathrm{~cm}$ sobre el nivel del suelo, por lo que ambas alturas presentaron el mismo efecto del crecimiento, el cual fue significativamente mayor con respecto al testigo en la época seca.

Finalmente se determinó que la densidad de siembra de 1000 árb ha-1 incidió negativamente en el crecimiento de la plantación y generó disminución del crecimiento hasta en un $20 \%$ con respecto a las densidades de 4445 y 2500 árb ha-1 que no mostraron diferencias entre sí.

Para fines prácticos, la especie L. macrophylla en las mismas condiciones del estudio presenta mejores comportamientos si se cultiva a 4445 o 2500 árb ha-1, con una poda ya sea a $40 \circ 100 \mathrm{~cm}$ y preferiblemente realizada en la época lluviosa.

\section{Recomendaciones}

Se recomienda reproducir el experimento con plantaciones de la misma especie con edades de tres a cinco años y considerando otras condiciones de sitio típicas de los lugares potenciales para el desarrollo de proyectos con fines energéticos.

Se recomienda evaluar variables como el Índice de área foliar, producción de biomasa, morfología de crecimiento y características calóricas como indicadores en los primeros dos meses ya que facilitarían evaluar a nivel de copa la calidad de los individuos; además, de evaluar la poda a $10 \mathrm{~cm}$ sobre nivel de suelo que permitiría cosechar volúmenes de biomasa mayores.

\section{Agradecimientos}

Se agradece a la UNACIFOR (Universidad Nacional de Ciencias Forestales), por todo el apoyo logístico y científico para el desarrollo de la investigación, a la Empresa INCAL S.A. de C.V. del Grupo CALIDRA, por su apoyo financiero para el establecimiento y evaluación de la plantación. Al personal del Laboratorio de Ecofisiología Forestal y aplicaciones ecosistémicas (ECOPLANT) del Tecnológico de Costa Rica por el apoyo en la redacción y afinamiento de esta publicación.

\section{Referencias}

[1] D. Pérez y M. Kanninen, «Heartwood, sapwood y bark content and wood specific gravity of young and mature Tectona grandis trees in Costa Rica,» Silva Fennica, vol. 37, $\mathrm{n}^{\circ} 1$, pp. 45-54, 2002.

[2] H. Lu, A. Hanandeh y M. Wi, «Assessment of bioenergy production from mid-rotation thinning of hardwood plantation: life cycle assessment and cost analysis,» Clean Techn Environ Policy, vol. 19, p. 2021-2040, 2017.

[3] L. Pancel, Basic outline of tree plantations in the tropics, Amsterdam: Springer, 2016.

[4] S. Brown, A. Gillespe y A. Lugo, «Biomass estimation for tropical forest with applications to forest inventory data," Forest Science, vol. 34, n 4, p. 881 - 902, 1989.

[5] R. Baettig, A. Yañez y A. Albornoz, Cultivos Dendroenergéticos de Híbridos de Alamo para la obtencion de biocombustibles en Chile: estado del arte, Universidad de Talca, Centro Técnologico del Alamo, Chile, 2010.

[6] CEPAL, «Istmo centroamericano: Las fuentes de energía renovable y elcumplimiento de la estrategia 2020,» 2009. [En línea]. Available: http://www.ceaconline.org/pdf/ CEPAL_FUENTES_RENOVABLES.pdf. [Último acceso: 2009 Agosto 1].

[7] COMMEND, «"LEAP software”. Community for energy, environment and development,» 2011. [En línea]. Available: http://www.energycommunity.org/. [Último acceso: 2017 Octubre 10].

[8] P. Aguilera, Diagnóstico del subsector hidrocarburos. En base de datos de DGE-Honduras., 2009.

[9] W. Flores, El sector energético de Honduras: Diagnóstico y política energética., San Pedro Sula, Honduras. 20p., 2012.

[10] E. Salazar-Zeledón, «Influencia de altas densidades de plantación en el poder calorífico y propiedades físicas de la madera parala especie Gmelina arborea Roxb. ex Sm,» Revista Forestal Mesoamericana Kurú, vol. 14, n 30, pp. 51-56., 2016. 
[11] Climate-Data.org, «Climate-Data.org,» 2012. [En línea]. Available: $\quad$ http://es.climate-data.org/location/32119/. [Último acceso: 2016 febrero 26].

[12] m. Dynesius, "Slow recovery of bryophyte assemblages in middle-aged boreal forests regrown after clear-cutting,» Biological Conservation, vol. 109, pp. 101-109, 2015.

[13] A. Singhala, M. Kumarb, M. Bhattacharyab, N. Kumaric, P. Kumar, J. Devendra, K. Chauhanalndu y S. Thakur, «Pretreatment of Leucaena leucocephala wood by acidified glycerol: optimization, severity index and correlation analysis,» Bioresource Technology, vol. 265, pp. 214-223, 2018.

[14] R. Nassara, F. T. Nermeen y M. Redac, «Active yeast extract counteracts the harmful effects of salinity stress on the growth of leucaena plant,» Scientia Horticulturae, vol. 30, pp. 61-67, 2015.

[15] F. Reda, S. Maximous y O. El-Kobisy, «Morphological and anatomical studies on leucaena (Leucaena leucocephala) plants grown under stress of different levels of salinity in irrigation water, B Bull. Fac. Agric. Cairo Univ, vol. 4, pp. 309330, 2000.

[16] L. Pancel, Basic outline of tree plantations, Amsterdam: Springer, 2016.

\section{Este artículo debe citarse como:}

Flores-Pinot, D., Janeth-Sorto, T., Gutiérrez-Bardales, J., Arias-Aguilar, D., Valverde, J. C., \& Mora- Molina, J. (2018). Capacidad de rebrote de Leucaena macrophylla Benth con fines dendroenergéticos en Cortes, Honduras. Revista Forestal Mesoamericana Kurú, 16(38), 47-54. Doi. 10.18845/rfmk.v16i38.3995 